\title{
Knowledge Management in the Cloud: Benefits and Risks
}

\author{
Mehmet Sabih AKSOY \\ College of Computer and Information Sciences \\ Department of Information Systems, \\ King Saud University \\ Kingdom of Saudi Arabia
}

\author{
Danah Algawiaz \\ Information Systems Department \\ Shaqra University \\ Kingdom of Saudi Arabia
}

\begin{abstract}
The success of organizations largely depends on continual investment in learning and acquiring new knowledge that creates new businesses and improve existing performance. So, using Knowledge management must result in better achieving, or even exceeding, organizations objectives. The purpose of knowledge management must not be to just become more knowledgeable, but to be able to create, transfer and apply knowledge with the purpose of better achieving objectives. As new technologies and paradigms emerge, businesses have to make new efforts to properly get aligned with them, especially in knowledge management area. Today the Cloud Computing paradigm is becoming more and more popular, due to the vast decrease in time, cost and effort for meeting software development needs. It also provides a great means for gathering and redistributing knowledge. In this paper, we will discuss the benefits and risks of using cloud computing in knowledge management systems.
\end{abstract}

Keywords: Knowledge, Cloud Computing, Knowledge Management, Service Oriented Architecture, Grid computing, Knowledge Management Systems.

\section{INTRODUCTION}

Knowledge is an understanding of someone or something, such as facts, information, descriptions, or skills, which is acquired through experience or education by perceiving, discovering, or learning [1]. Also, knowledge can refer to a theoretical or practical understanding of a subject. Furthermore, it can be implicit or explicit [2]. Explicit knowledge is knowledge that has been articulated, codified, and stored in certain media. Moreover, it can be readily transmitted to others. However, tacit knowledge is the kind of knowledge that is difficult to transfer [3]. A knowledge management system is most often used in business in applications such as information systems, business administration, computer science, public policy and general management. Organizations need Knowledge Management for finding, mapping, gathering, filtering information, developing new knowledge, converting personal knowledge into shared knowledge resources, understanding and learning, and adding value to information to create knowledge. The three fundamental processes of knowledge management are knowledge acquisition, knowledge sharing, and knowledge utilization [4]. However, Knowledge management is not a static process, it is dynamic from two dimensions: the business and the technology [5]. So, for the success of knowledge management, it should be kept aligned with the business and the technology, which is fast upgrading. One of the new popular technological paradigms is cloud computing (CC) that is an extension to grid computing and the serviceoriented architecture (SOA) [6]. As [6] articulates, CC has five key characteristics: providing on demand self-service, based on broad network access, making advantage of resource pooling, rapid elasticity based on cloud consumers' resource needs, and providing the ability to measure the provided services. In this paper, we aim to discuss the benefits and risks of using cloud computing in knowledge management systems. The reminder of this article is organized as follows: section 2 is devoted to explain knowledge Management Systems. However section 3 will explain cloud computing. Benefits of Using Cloud Computing in Knowledge Management systems are detailed in section 4 . Then section 5 will explain Risks of Using Cloud Computing in Knowledge Management Systems. Finally we conclude in section 6 .

\section{KNOWLEDGE MANAGEMENT SYSTEMS}

It is not easy to define the term "knowledge" as it has different meanings depending on context. In the context of the business enterprise or the personal computer user, knowledge tends to connote possession of experienced "know-how" as well as possession of factual information or where to get it [1]. In philosophy, the theory of knowledge is called epistemology and deals with such questions as how much knowledge comes from experience or from innate reasoning ability; whether knowledge needs to be believed or can simply be used; and how knowledge changes as new ideas about the same set of facts arise [1]. Knowledge management is A method for the improvement of business process performance [3]. In addition, a knowledge management system is most often used in business in applications such as information systems, business administration, computer science, public policy and general management. Also, common company departments for knowledge management systems include human resources, business strategy and information technology. Moreover, knowledge management systems consist of processes to capture, distribute, and effectively use knowledge [7]. Knowledge acquisition is the process of development and creation of insights, skills, and relationships [4]. On the other hand, Knowledge sharing is disseminating and making available what is already known. However, Knowledge utilization includes research, scholarly, and programmatic intervention activities aimed at increasing the use of knowledge to solve problems [8]. The knowledge Management functions are finding, mapping, gathering, filtering information, developing new knowledge, converting personal knowledge into shared knowledge resources, understanding and learning, adding value to information to create knowledge, enabling action through knowledge, processing shared knowledge resources, delivering explicit 
knowledge, and building adequate technical infrastructures [9].

\section{CLOUD COMPUTING}

Cloud Computing is an extension to grid computing and the Service-Oriented Architecture [6]. And Grid computing is the collection of computer resources from multiple locations to reach a common goal. Also, Grid computing is applying the resources of many computers in a network to a single problem at the same time - usually to a scientific or technical problem that requires a great number of computer processing cycles or access to large amounts of data [10]. Service-oriented architecture (SOA) is a software design and software architecture design pattern based on distinct pieces of software providing application functionality as services to other applications [6]. Moreover, this is known as serviceorientation. In addition, it is independent of any vendor, product or technology [10]. Service-Oriented Architecture makes it easy for computers connected over a network to cooperate [6]. Besides, every computer can run an arbitrary number of services, and each service is built in a way that ensures that the service can exchange information with any other service in the network without human interaction and without the need to make changes to the underlying program itself [6]. Cloud computing is internet-based computing in which large groups of remote servers are networked to allow the centralized data storage, and online access to computer services or resources. Furthermore, clouds can be classified as public, private, community or hybrid [9]. Private cloud is accessible from an intranet, internally hosted, and used by a single organization [11]. Community cloud has infrastructure accessible to a specific community. And public cloud is accessible from the internet, externally hosted, and used by the general public [12]. Finally, hybrid cloud is a combination of two or more clouds [13]. Moreover, there are five essential characteristics of cloud computing: on-demand self-service, broad network access, resource pooling, rapid elasticity, and measured service [14]. Users of On-demand self-service are able to provision cloud computing resources without requiring human interaction, mostly done though a web-based selfservice portal (management console) [15]. In contrast, broad network access means that cloud computing resources are accessible over the network, supporting heterogeneous client platforms such as mobile devices and workstations [15]. But resource pooling is the service multiple customers from the same physical resources, by securely separating the resourceson logical level [15]. And rapid elasticity is the resources that provisioned and released on-demand and/or automated based on triggers or parameters [15]. Resource usage are monitored, measured, and reported (billed) transparently based on utilization by measured service [16]. Furthermore, cloud computing is much more than just virtualization. It's really about utilizing technology "as a service". There are three Service models of cloud computing: Infrastructure as a service, Platform as a service, and Software as a service [17]. Infrastructure as a service (IaaS) provides access to server hardware, storage, network capacity, and other fundamental computing resources [17]. And Platform as a service (PaaS) provides access to basic operating software and services to develop and use customer-created software applications [18]. Finally, Software as a service (SaaS) provides integrated access to a provider's software applications [17].

\section{BENEFITS OF USING CLOUD COMPUTING IN KNOWLEDGE MANAGEMENT SYSTEMS}

Cloud computing provides a scalable online environment that makes it possible to handle an increased volume of work without impacting system performance. Cloud computing also offers significant computing capability and economy of scale that might not otherwise be affordable, particularly for small and medium-sized organizations, without the IT infrastructure investment [19]. Organizations can provide unique services using large-scale computing resources from cloud service providers, and then nimbly add or remove IT capacity to meet peak and fluctuating service demands while only paying for actual capacity used. Moreover, organizations can rent added server space for a few hours at a time rather than maintain proprietary servers without worrying about upgrading their resources whenever a new application version is available [20]. They also have the flexibility to host their virtual IT infrastructure in locations offering the lowest cost. Optimized IT infrastructure provides quick access to needed computing services. In addition, providing the right level of security for knowledge management system is a challenging that can be solved by using cloud computing. Also, cloud computing can keep knowledge management up with technology. By using private cloud sensitive information should be shielded from most users, while allowing easy access to those with the proper credentials. Furthermore, community and hybrid cloud can motivated people and overcoming organizational culture challenges by developing a culture that embraces learning, sharing, changing, and improving knowledge sharing [19].

\section{RISKS OF USING CLOUD COMPUTING IN KNOWLEDGE MANAGEMENT SYSTEMS}

Depending on the cloud solution used (SaaS, PaaS, or IaaS), users of knowledge management system may be unable to obtain and review network operations or security incident logs. A multi-tenant cloud environment in which users of knowledge management system and applications share resources presents a risk of data leakage that does not exist when dedicated servers and resources are used exclusively by one organization [21]. Also, if cloud computing is adopted to a significant degree, an organization needs fewer internal IT personnel in the areas of infrastructure management, technology deployment, application development, and maintenance. So, the morale and dedication of remaining IT staff members could be at risk as a result. Many cloud service providers are relatively young companies, or the cloud computing business line is a new one for a well-established company. Hence the projected longevity and profitability of cloud services are unknown. At the time of publication, some Cloud Service Providers are curtailing their cloud service offerings because they are not profitable. And cloud computing service providers might eventually go through a consolidation period. As a result, Cloud Service Provider customers might face operational disruptions or incur the time and expense of researching and adopting an alternative solution, such as converting back to in-house hosted solutions [21]. 


\section{CONCLUSION}

Using Knowledge management systems must result in better achieving, or even exceeding, organizations objectives. However, Knowledge management is not a static process; it is dynamic from two dimensions: the business and the technology. And as new technologies and paradigms emerge, businesses have to make new efforts to properly get aligned with them, especially in knowledge management area. Today the Cloud Computing paradigm is becoming more and more popular, due to the vast decrease in time, cost and effort for meeting software development needs. It also provides a great means for gathering and redistributing knowledge. But using cloud computing in Knowledge management system has some risks like risk of data leakage, IT organizational changes and cloud service provider viability. Our future work will be a bout decrease risks of using cloud computing in knowledge management systems by reducing likelihood and impact of risks in cloud computing.

\section{REFERENCES}

[1] Stanley Cavell, "Knowing and Acknowledging", Must We Mean What We Say? (Cambridge University Press, 2002), 238-266.

[2] Drucker, P., The age of discontinuity: guidelines for our changing society. New York: Harper \& Row, 1969.

[3] Davenport, Thomas H. (1994). "Saving IT's Soul: Human Centered Information Management". Harvard Business Review 72 (2): 119-131.

[4] Alavi, M. and Leidner, D., Review: knowledge management and knowledge management systems: conceptual foundations and research issues, MIS Quarterly, Vol. 25 No. 1, pp. 107-36, 2001.

[5] Gupta, Jatinder; Sharma, Sushil (2004). Creating Knowledge Based Organizations. Boston: Idea Group Publishing. ISBN 1-59140-163-1.

[6] P. Mell, and T. Grance, Draft NIST Working Definition of Cloud Computing, 2009.

[7] Wong, K.Y. and E. Aspinwall; Characterizing knowledge management in the small business environment, Journal of Knowledge Management, vol. 8, pp. 44-61, 2004.

[8] Jay Liebowitz, Strategic Intelligence: Business Intelligence, Competitive Intelligence, and Knowledge Management, Auerbach Publications, 2006.

[9] Maier, R., Knowledge management systems, 3rd edition, Springer, 2007.

[10] I. Sriram and A. Khajeh-hosseini, Research Agenda in Cloud Technologies, Methodology abs/1001.3, 2010.

[11] Foley, John. "Private Clouds Take Shape". InformationWeek. Retrieved 2010-08-22

[12] Rouse, Margaret. "What is public cloud?". Definition from Whatis.com. Retrieved 12 October 2014.

[13] Rouse, Margaret. "What is a multi-cloud strategy". SearchCloudApplications. Retrieved 3 July 2014.

[14] "What is Cloud Computing?". Amazon Web Services. 2013-03-19. Retrieved 2013-03-20.

[15] "Defining 'Cloud Services' and "Cloud Computing"'. IDC. 2008-09-23. Retrieved 2010-08-22.

[16] Danielson, Krissi (2008-03-26). "Distinguishing Cloud Computing from Utility Computing". Ebizq.net. Retrieved 2010-08-22.
[17] Keep an eye on cloud computing, Amy Schurr, Network World, 2008-07-08, citing the Gartner report, "Cloud Computing Confusion Leads to Opportunity". Retrieved 2009-09-11.

[18] Boniface, M. et al. (2010), Platform-as-a-Service Architecture for Real-Time Quality of Service Management in Clouds, 5th International Conference on Internet and Web Applications and Services (ICIW), Barcelona, Spain: IEEE, pp. 155-160, doi:10.1109/ICIW.2010.91

[19] "Towards Continuous Cloud Service Assurance for Critical Infrastructure IT". The 2nd International Conference on Future Internet of Things and Cloud (IEEE FiCloud-2014). Retrieved 2014-08-15.

[20] Kemal A. Delic, Jeff A. Riley, Enterprise Knowledge Clouds: Next Generation KM Systems?, International Conference on Information, Process, and Knowledge Management, 2009.

[21] Albena Antonova, Roumen Nikolov, Conceptual KMS Architecture within Enterprise 2.0 and Cloud Computing, Computing, 2005. 